\title{
ALGORITHM FOR THE AUTOMATIC ESTIMATION OF AGRICULTURAL TREE GEOMETRIC PARAMETERS USING AIRBORNE LASER SCANNING DATA
}

\author{
E. Hadaś ${ }^{\mathrm{a}, *}$, A. Borkowski ${ }^{\text {a }}$, J. Estornell $^{\text {b }}$ \\ ${ }^{\text {a }}$ Wrocław University of Environmental and Life Sciences, Institute of Geodesy and Geoinformatics, Poland - (edyta.hadas, \\ andrzej.borkowski)@igig.up.wroc.pl \\ ${ }^{\mathrm{b}}$ Universitat Politècnica de València, Department of Cartographic Engineering, Geodesy and Photogrammetry, Spain - \\ jaescre@cgf.upv.es
}

Commission VIII, WG VIII/7

KEY WORDS: ALS, trees, agriculture, $\alpha$-shape, PCA, algorithms

\begin{abstract}
:
The estimation of dendrometric parameters has become an important issue for the agricultural planning and management. Since the classical field measurements are time consuming and inefficient, Airborne Laser Scanning (ALS) data can be used for this purpose. Point clouds acquired for orchard areas allow to determine orchard structures and geometric parameters of individual trees. In this research we propose an automatic method that allows to determine geometric parameters of individual olive trees using ALS data. The method is based on the $\alpha$-shape algorithm applied for normalized point clouds. The algorithm returns polygons representing crown shapes. For points located inside each polygon, we select the maximum height and the minimum height and then we estimate the tree height and the crown base height. We use the first two components of the Principal Component Analysis (PCA) as the estimators for crown diameters. The $\alpha$-shape algorithm requires to define the radius parameter $R$. In this study we investigated how sensitive are the results to the radius size, by comparing the results obtained with various settings of the $R$ with reference values of estimated parameters from field measurements. Our study area was the olive orchard located in the Castellon Province, Spain. We used a set of ALS data with an average density of 4 points $\mathrm{m}^{-2}$. We noticed, that there was a narrow range of the $R$ parameter, from $0.48 \mathrm{~m}$ to $0.80 \mathrm{~m}$, for which all trees were detected and for which we obtained a high correlation coefficient $(>0.9)$ between estimated and measured values. We compared our estimates with field measurements. The RMSE of differences was $0.8 \mathrm{~m}$ for the tree height, $0.5 \mathrm{~m}$ for the crown base height, $0.6 \mathrm{~m}$ and $0.4 \mathrm{~m}$ for the longest and shorter crown diameter, respectively. The accuracy obtained with the method is thus sufficient for agricultural applications.
\end{abstract}

\section{INTRODUCTION}

The reliable knowledge of tree parameters like tree height, crown base height and crown shape, allows to efficiently manage and plan the agricultural production (Doruska and Burkhart, 1994). These parameters cannot be easily obtained for large areas with classical dendrometric measurements, as these methods are very time consuming. Due to an increased availability of Airborne Laser Scanning (ALS) data and decreasing costs of performing ALS flights e.g. with unmanned aerial vehicle (UAV), it is desired to develop automatic methods and algorithms of estimating abovementioned parameters from ALS data.

The vast majority of research in the field of remote sensing of tree geometric parameters is devoted to the forest inventory (Hyyppä et al., 2012). Methods of acquiring dendrometric characteristics over large area of forests have been under development for more than a decade. The research started from determining forest terrain elevations (Kraus and Pfeifer, 1998), through the base area approach (Holopainen et al., 2010), while current developments allows identification of a single tree (Vauhkonen et al., 2011) and crown delineation (Dalponte et al., 2015). ALS data are commonly used for the estimation of tree heights (Saremi et al., 2014), crown base heights (Vauhkonen, 2010), crown dimensions (Popescu and Zhao,
2008). These parameters can be used for the indirect estimation of the biomass volume (Hauglin et al., 2013).

For agricultural areas, the potential of ALS data for management and production seems to be still unexploited and not well investigated. There are two main differences between forest and agricultural trees. Forest trees are usually close to each other, supressed, with overlapping crowns, while the agricultural trees are kept at a distance, due to artificial planting and regular pruning. Secondly, a significant fraction of the woody biomass of forest trees is concentrated in trunk, so a special attention is paid for trunk parameters. Agricultural trees have usually short stem and the biomass is concentrated in the crown of a plant, so crown characteristics are of main interests (Estornell et al., 2014). Because of the abovementioned differences, it should be investigated whether the methods and algorithms already developed in forestry are suitable for agricultural studies.

In this study we used ALS data with density of 4 points $\mathrm{m}^{-2}$ covering olive orchard located in Spain. We applied the $\alpha$-shape algorithm to normalized point cloud to obtain crown contours and perform analysis on ALS points located inside the crown in order to estimate tree height, crown base height and crown diameters. We tested various setting of the radius $R$ ( $\alpha$-shape input parameter) to select the optimum value of $R$ for our test

* Corresponding author 
area. We demonstrate the impact of $R$ on crown shape delineation and final estimates of tree geometric parameters. Finally we present the comparison of estimated parameters with field measurements for 25 sampled trees. This paper is divided into four main parts. After the introduction, in section 2 we describe methodology and data. Section 3 describe our results and section 4 contains conclusions and recommendations for future studies.

\section{METHODS AND DATA}

\subsection{Original algorithm}

The proposed method of automatic estimation of tree geometric parameters of agricultural trees from ALS data is based on the $\alpha$-shape algorithm. The algorithm should operate on the normalized ALS data, so a digital terrain model (DTM) of corresponding area should be generated from ALS points classified as ground reflections. Ground reflections should then be removed from the normalized ALS data for further processing.

In the first stage, tree crowns are delineated with $\alpha$-shape algorithm. The $\alpha$-shape algorithm (Edelsbrunner et al., 1983) is an approach to describe the shape of a finite set of points on a plane. The $\alpha$-shape output for a defined radius $R$ is a set of contours, defining the boundaries of objects that are represented by a set of points with heterogeneous distribution and low density. The crucial step of applying the $\alpha$-shape algorithm successfully is to define the value of $R$ parameter accordingly to the characteristics of the data. After the $\alpha$-shape algorithm is performed, some tree geometric parameters can be estimated using the ALS points detected inside each of the delineated tree crown. Tree height is estimated as a height of the highest point in the sample, crown base height as a height of the lowest point in the sample. Then the Principal Component Analysis (PCA) algorithm (Jolliffe, 1986) can be performed on the sample to estimate crown diameters: the longest one and the perpendicular to the longest one (referred further in the paper as the shorter diameter).

\subsection{Source data}

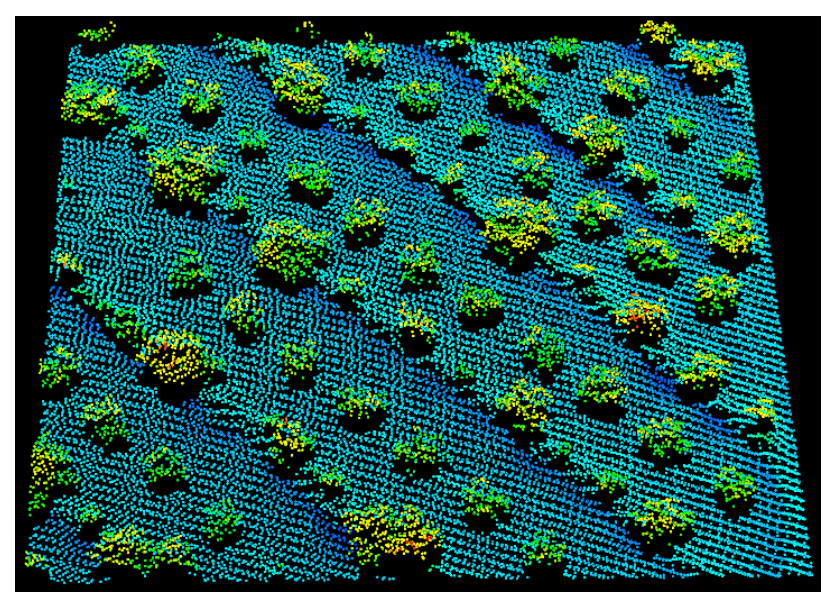

Figure 1. Plot of ALS point cloud of a part of the study area

For this study, we used the airborne laser scanning data collected in September 2012 with Leica ALS50-II instrument, with $1500 \mathrm{~m}$ of average flight height. The average density of the obtained point cloud was 4 points $/ \mathrm{m}^{2}$. The point cloud covers a part of olive orchard located in the West Central part of Spain, close to the city of Valencia. The area of the test site is 5.92 ha. We also used an orthophoto in near-infrared spectrum that was available with a nominal resolution of $0.5 \mathrm{~m}$ from CInstitut Cartogràfic Valencià of the Valencia region, Spain. The ortophoto was used as a source of approximated crown tree centres and for visual assessment of the results.

\subsection{Reference data}

In order to obtain reference data for tree geometric parameters, 25 trees were selected inside the study area and classical dendrology measurements were performed to obtain tree height, crown base height, stem diameter, length of the longest diameter and length of shorter diameter. We consider the sampled trees to be a representative set of all trees in the orchard. Tree heights varies from $3.12 \mathrm{~m}$ to $4.48 \mathrm{~m}$, crown base height varies from $0.60 \mathrm{~m}$ to $1.07 \mathrm{~m}$, lengths of the longest diameters varies from $3.16 \mathrm{~m}$ to $6.86 \mathrm{~m}$ and lengths of shorter diameter varies from $2.71 \mathrm{~m}$ to $5.89 \mathrm{~m}$. Moreover, the GPS-RTK measurements of tens of ground points were performed to check the accuracy of the DTM used in point cloud normalization.

\subsection{Methodology}

Using the ALS data, we created a DTM using ArcGIS 10 software as a $4 \times 4 \mathrm{~m}$ grid, assigning each cell an average height of points located inside the cell and classified as ground points. Then, we normalized the point cloud by subtracting DTM heights from ALS point heights, obtaining thus height points from the ground. We removed all points from ALS normalized dataset having heights below $0.5 \mathrm{~m}$ to filter out ground points and points reflected from low vegetation. We implemented the $\alpha$-shape algorithm and PCA algorithm in Matlab environment and performed numerical experiments on the ALS data, by changing the value of $R$ within the range of $0.4 \mathrm{~m}$ to $2.5 \mathrm{~m}$, with a step of $0.02 \mathrm{~m}$. In each run of the algorithm, we estimated tree height, crown base height, crown diameters and crown shape area. We compared the estimated values with field measurements and calculated correlation coefficients between estimated and reference values.

\section{RESULTS}

\subsection{Impact of radius value on $\alpha$-shape algorithm results}

We noticed that the crown contours obtained with $\alpha$-shape algorithm are sensitive to the value of $R$ (see Figure 2). For small $R$, single trees were usually covered by more than one polygon and crown contours were unnaturally irregular and sharped. Moreover, not all points that truly belong to tree crown were detected as crown points. On the other hand, for large $R$ trees were often merged to each other, so that a single polygon included more than a single tree inside. We noticed there was only a narrow range of $R$ value for which trees were correctly identified, separated and their crown contours were regular.

We investigated the influence of the $R$ value on the percentage of correctly detected trees and the correlation coefficient between estimated tree geometric parameters and field measurements (see in Figure 3). Tree was considered to be correctly detected if its crown shape polygon included ALS points of a single tree only and tree centroid obtained from the ortophoto was inside the polygon. The correlation coefficient was calculated only including estimates from correctly detected trees. 

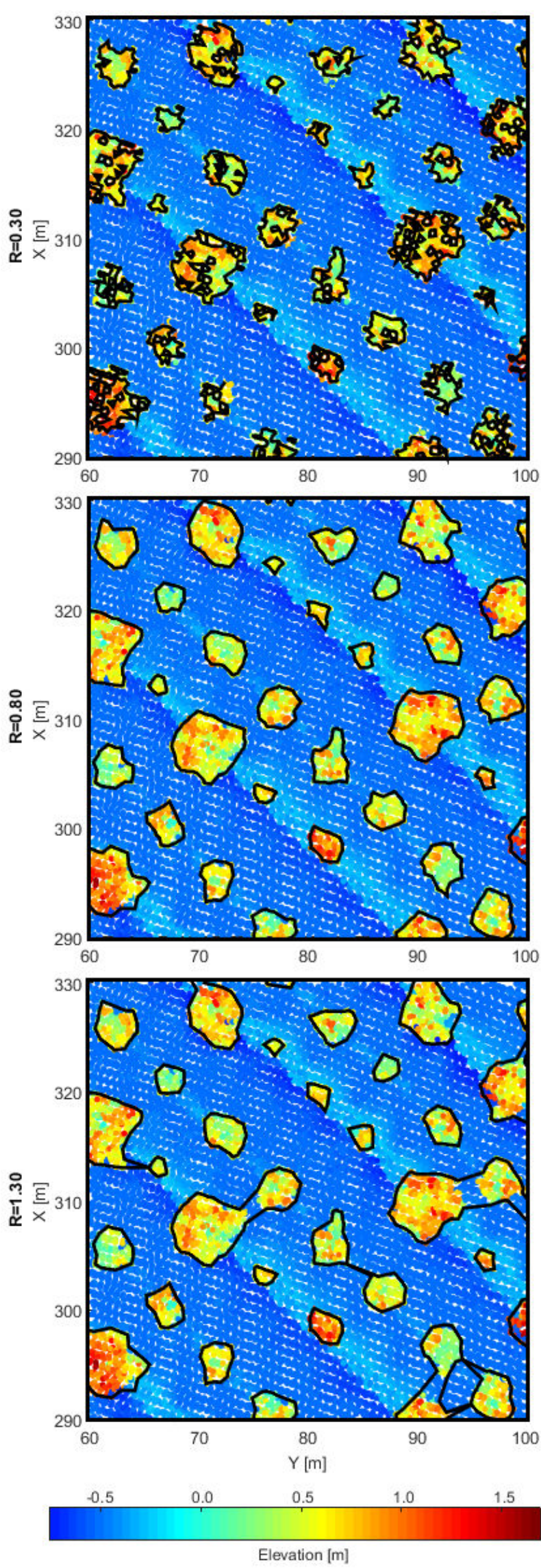

Figure 2. Delineated tree crown shapes for a part of study area for exemplary value of $R$; point cloud in the background coloured by point elevation

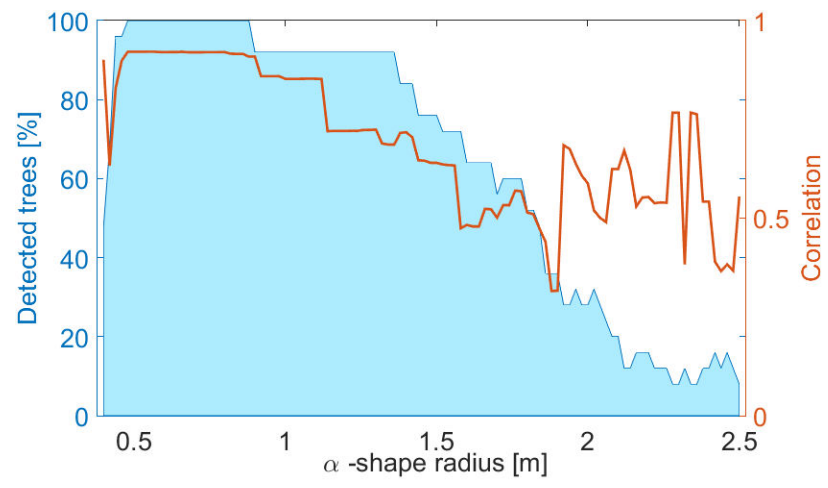

Figure 3. $R$ value impact on percentage of detected trees and correlation coefficient between estimated and measured tree geometric parameters

As expected, we noticed a narrow range of $R$ value, for which $100 \%$ of trees were detected correctly. For small $R$, not all trees were detected, and for $R<0.40 \mathrm{~m}$ a huge amount of very small polygons were obtained and no tree was identified correctly so these results are not available in Figure 3. For $R>0.80 \mathrm{~m}$ the number of correctly detected trees decreased by 2 up to $R=1.36$ $\mathrm{m}$, and then it was decreasing consequently up to less than $20 \%$ of detected trees for $R>2.10 \mathrm{~m}$.

At the same time, the calculated correlation coefficient was also very high $(>0.9)$ when $100 \%$ of trees were detected. The highest value of correlation coefficient $(>0.92)$ was obtained for $R$ between $0.48 \mathrm{~m}$ and $0.80 \mathrm{~m}$. For $R>0.80 \mathrm{~m}$ the correlation coefficient decreased significantly, with a minimum of 0.31 for $\mathrm{R}=1.88 \mathrm{~m}$. For $\mathrm{R}>1.90 \mathrm{~m}$ the correlation coefficient varies significantly between 0.36 to 0.77 , depending on the number of detected trees. These high values of correlation coefficient for large $R$ can be explained by the fact, that only estimates from correctly detected trees were included in the calculations, and for large $R$ some isolated trees could still be detected correctly.

\subsection{Quality assessment of the results}

To assess the quality of tree geometric parameters estimated with the approach based on a-shape algorithm, we compared our results obtained for $R=0.80 \mathrm{~m}$ with field measurements. We chose $R=0.80 \mathrm{~m}$, because this was the highest value of $R$ for which the correlation coefficient was still larger than 0.92 and $100 \%$ of sampled trees were detected correctly. The high value of $R$ allows to minimize the number of artefacts (very small polygons). Crown shapes estimated with $R=0.80 \mathrm{~m}$ for a part of study area are presented in Figure 2 (middle plot).

The comparison of estimated tree height, crown base height, crown diameters and crown area with field measurements are presented in Figure 4. It is important to note, that crown shape areas were not subject of direct field measurements, but it was calculated as an area of ellipse defined by two measured perpendicular diameters, so this comparison is only presented for rough verification.

Our tree height estimates were always smaller than reference values, with mean difference of $-0.7 \mathrm{~m}$ and standard deviation of $0.3 \mathrm{~m}$. In the contrary, crown base heights were always higher than reference values, with mean difference of $0.4 \mathrm{~m}$ and standard deviation of $0.2 \mathrm{~m}$ This means that the estimated crowns were flattened. Crown shape diameters were usually overestimated. The mean difference was $0.4 \mathrm{~m}$ for the longest diameter and $0.2 \mathrm{~m}$ for shorter diameter. Standard deviation was 
0.4 and 0.5 respectively. The majority of differences for both diameters was below $0.5 \mathrm{~m}$. Estimated crown shape areas varies from the ellipse areas up to $10.5 \mathrm{~m}^{2}$, but only for 4 trees this difference was larger than $3 \mathrm{~m}^{2}$. The mean difference was -1.0 $\mathrm{m}^{2}$ and standard deviation was $3.1 \mathrm{~m}^{2}$.
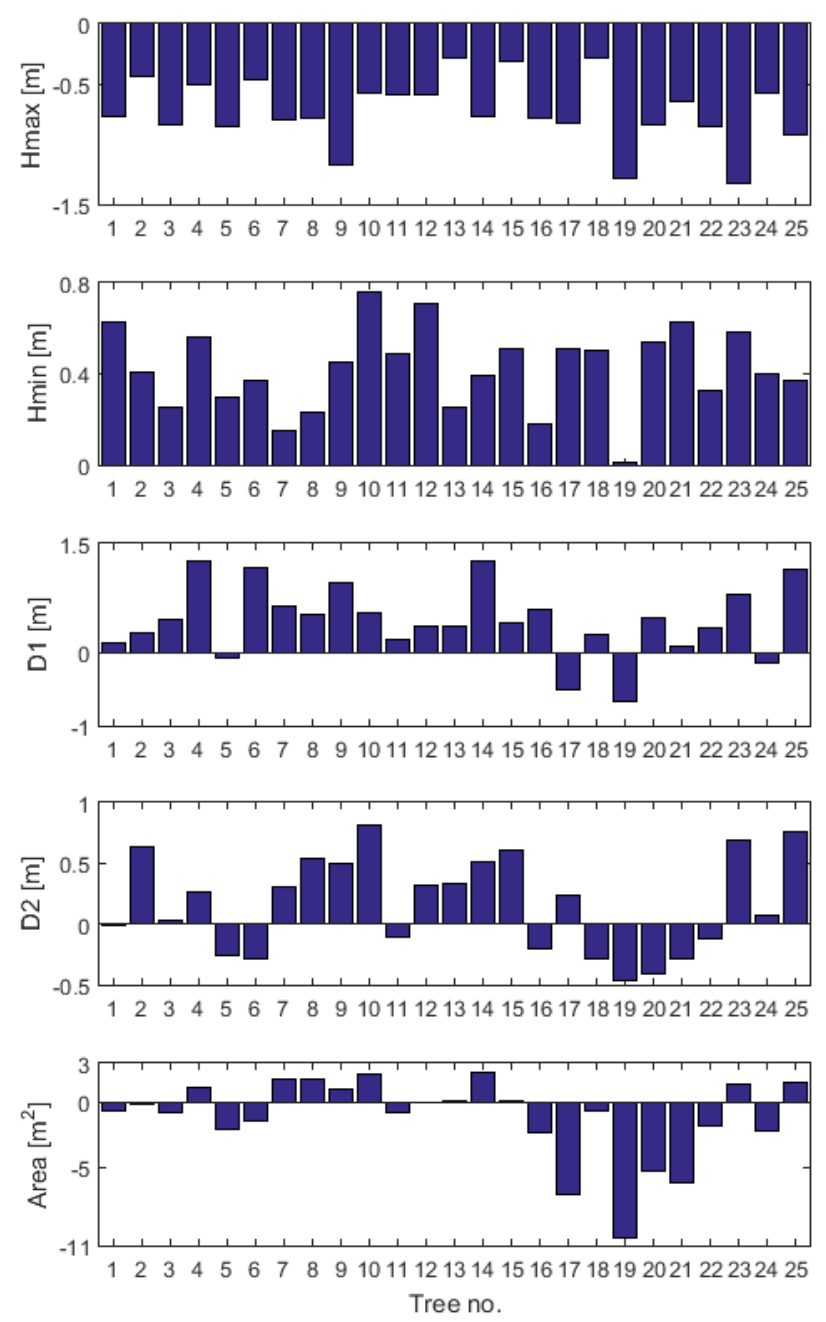

Figure 4. Differences between estimated tree geometric parameters and field measurements

\section{CONCLUSIONS}

We proposed a methodology to estimate selected geometric parameters of agricultural trees. This methodology is based on $\alpha$-shape algorithm, that requires to define the value of radius $R$. We investigated the impact of $\mathrm{R}$ value on final estimates and noticed, there is only a narrow range of $\mathrm{R}$ for which all trees are detected correctly. The RMSE of differences between estimated parameters and field measurements was $0.8 \mathrm{~m}$ for the tree height, $0.5 \mathrm{~m}$ for the crown base height, $0.6 \mathrm{~m}$ and $0.4 \mathrm{~m}$ for the longest and shorter crown diameter respectively. The accuracy obtained with the method is thus sufficient for agricultural applications.

The research we present are a part of undertaken studies on automatic estimation of geometric parameters of agricultural trees. Several further experiments are under investigation, including the automatic $R$ value adjustment based on classical field measurements of representative trees in the study area.

\section{REFERENCES}

Dalponte, M., Reyes, F., Kandare, K., Gianelle, D., 2015. Delineation of Individual Tree Crowns from ALS and Hyperspectral data: a comparison among four methods. European Journal of Remote Sensing, 48, pp. 365-382.

Doruska, P., Burkhart H., 1994. Modeling the diameter and locational distribution of branches within the crows of loblolly pine trees in unthinned plantations. Canadian Journal of Forest Research, 24, pp. 2362-2376.

Edelsbrunner, H., Kirkpatrick, D.G., Seidel, R., 1983. On the shape of a set of points in the plane. IEEE Transactions on Information Theory, 29(4), pp. 551-559.

Estornell, J.,Velázquez-Marti, B., López-Cortés, I., Salazar, D., Fernández-Sarría, A., 2014. Estimation of wood volume and height of olive tree plantations using airborne discrete-return LiDAR data. GIScience \& Remote Sensing, 51(1), pp. 17-29

Hauglin, M., Dibdiakova, J., Gobakken, T., Næsset, E., 2013. Estimating single-tree branch biomass of Norway spruce by airborne laser scanning. ISPRS Journal of Photogrammetry and Remote Sensing, 79, pp. 147-156.

Holopainen, M., Vastaranta, M., Rasinmäki, J., Kalliovirta, J., Mäkinen, A., Haapanen, R., Melkas, T., Yu, X., Hyyppä, J., 2010. Uncertainty in timber assortment estimates predicted from forest inventory data. European Journal of Forest Research, 129, pp. 1131-1142.

Hyyppä, J., Yu, X., Hyyppä, H., Vastaranta, M., Holopainen, M., Kukko, A., Kaartinen, H., Jaakkola, A., Vaaja, M., Koskinen, J., Alho, P., 2012. Advances in Forest Inventory Using Airborne Laser Scanning. Remote Sensing, 4(5), pp. 1190-1207.

Jolliffe, I.T., 1986. Principal Component Analysis. SpringerVerlag, pp. 487

Kraus, K., Pfeifer, N., 1998. Determination of terrain models in wooded areas with airborne laser scanner data. ISPRS Journal of Photogrammetry and Remote Sensing, 53, pp. 193-203.

Popescu, S.C., Zhao, K., 2008. A voxel-based lidar method for estimating crown base height for deciduous and pine trees. Remote Sensing of Environment, 112(3), pp. 767-781.

Saremi, H., Kumar, L., Stone, C., Melville, G., Turner, R., 2014. Sub-compartment variation in tree height, stem diameter and stocking in a Pinus radiata D. Don Plantation examined using airborne LiDAR data. Remote Sensing, 6 (8), pp. 75927609 .

Vauhkonen, J., 2010. Estimating crown base height for Scots pine by means of the 3D geometry of airborne laser scanning data. International Journal of Remote Sensing, 31(5), pp. 12131226.

Vauhkonen, J., Ene, L., Gupta, S., Heinzel, J., Holmgren, J., Pitkänen, J., Solberg, S., Wang, Y., Weinacker, H., Hauglin, K., Lien, V., Packalén, P., Gobakken, T., Koch, B., Næsset, E., Tokola, T., Maltamo, M., 2011. Comparative testing of singletree detection algorithms under different types of forest. Forestry, 85(1), pp. 27-40. 